\title{
A Modular Robotic System for Ultrasound Image Acquisition
}

\author{
Randal P. Goldberg ${ }^{1}$, Mazilu Dumitru ${ }^{2}$, Russell H. Taylor ${ }^{1,3}$, Dan Stoianovici ${ }^{1,2}$ \\ 1 Mechanical Engineering Department, The Johns Hopkins University, Baltimore, MD, \\ USA \\ 2 Brady Urological Institute, Johns Hopkins Hospital, Baltimore, MD, USA \\ 3 Computer Science Department, The Johns Hopkins University, Baltimore, MD, USA
}

\begin{abstract}
This paper reports a modular robotic system currently in development for performing intra-operative 3-D ultrasound scans. The final goal is to be able to utilize ultrasound as a guidance and imaging modality for a variety of computer integrated surgery applications by overcoming the limits it's handheld nature presents. The primary component of the system is a compact 3 DOF robot called the Translational Remote Center of Motion Robot (TRCM). This unit, which can be used standalone or as a dexterity end effector for a larger degree of freedom base robot, can perform the motions necessary to locate and perform force controlled scans of target anatomy quickly and safely. This relatively inexpensive unit, while designed with the ultrasound application in mind, also becomes a useful general purpose robot for any application in which an RCM mechanism is desired.
\end{abstract}

\section{Introduction}

Ultrasound is a useful imaging tool for surgeons, capable of providing both 2-D images as well as 3-D models of the anatomy. These scans can be performed intraoperatively to provide real time information to the surgeon both about the target anatomy, as well as the location of surgical tools (such as needles in percutaneous therapy).

A major roadblock to the incorporation of ultrasound imaging in CIS applications is that it utilizes a handheld scanner to acquire the images. It is useful for giving the surgeon qualitative information about the anatomy, such as whether a tumor has grown or shrank, or in general where an instrument is relative to the anatomy is. Unfortunately, the images reflect both movements of the probe by the operator, as well as changes to the anatomy. In addition, there are many other operator dependant variables associated with ultrasound, such as variable scan times and inconsistent image quality due to changes in the applied force. These makes it difficult to perform the quantitative image processing necessary for it to be used as an effective tool to guide computer integrated surgery applications.

Several robotically assisted ultrasound systems have been developed, though not with the express purpose of intra-operative scanning. [1,2] In addition, they rely on large, specialized high degree of freedom robots to manipulate the ultrasound probe. 


\section{Robot Design}

A set of design requirements for the system was developed through conversations with members of the Johns Hopkins Hospital Ultrasonography department. It was decided that the ability to generate three target motions were desired: rotation and fan type scans, and translation for tracking patient motion and force control. (Figure 1) In addition, the range of motion for each of these degrees of freedom was determined from sonographer requests. Clearly rotation type models require $180^{\circ}$ of motion to complete. For the remaining two degrees of freedom, observation of and discussion with the clinicians led to a target of $120^{\circ}$ of motion minimum for the fan type scan, and $50 \mathrm{~mm}$ minimum of translational motion.

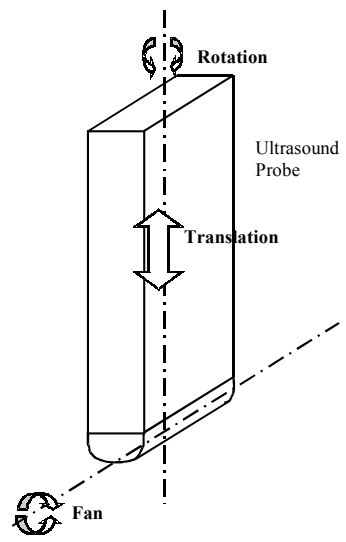

Figure 1: Desired Robot Degrees of Freedom

In addition, a simple experiment was performed with an instrumented ultrasound probe, to get a range of values for the forces the robot would encounter during a scan. Over a variety of scans, the sonographer produced an average of $7 \mathrm{~N}$ of force. This value agrees with the more complete evaluation reported in [1], and was used as a starting point for the translation stage design.

The two rotational motions lend themselves to RCM type designs, so a number of these were evaluated. Based on range of motion requirements, and the desire for a compact design, the mini "RCM" configuration was selected. [3] The mini "RCM" uses a chain drive to implement a parallelogram mechanism for it's pitch stage. For the ultrasound application, this degree of freedom actuates the fan type scan, and is

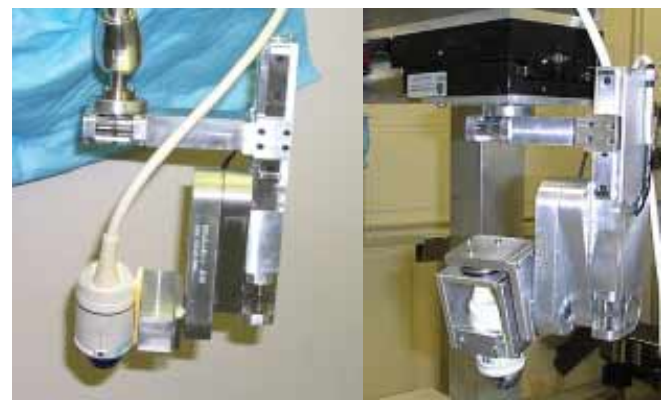

Figure 2: TRCM with US Probe on Passive Arm (left) and on a 3DOF cartesian base robot(right) adapted directly from the standard robot.

The difficulty in using the mini "RCM" for ultrasound arose from the size of the tool and the required range of motion. When the ultrasound probe is mounted on the robot, with it's face centered at the RCM point, it interferes with the pitch degree of freedom, limiting its range of motion. To overcome this, the roll stage of the robot was offset. This allows the ultrasound probe (and other large instruments) to be mounted at the RCM point, while maintaining full $360^{\circ}$ motion in both rotation joints.

The adjustable angle joint, which in the mini "RCM" allows tools to be placed at the RCM point, was removed, and in its place a translation stage was inserted. The 
translation stage uses a miniature ball screw actuator, and runs on a pair of recirculating linear ball bearings.

This new version of the RCM has been dubbed the Translational RCM (TRCM). In addition, it features a number of improvements over previous mini "RCM" versions. These include incorporation of high resolution redundant encoders on all three axes and a home sensor for zeroing the pitch stage. In addition, the TRCM utilizes newly developed ball worm drives ${ }^{4}$ in each of the rotation stages. The use of ball type drives on each axis makes the robot non-back drivable and very stiff with zero backlash.

\section{Preliminary and Future Work}

The prototype TRCM has been constructed, and is shown in Figure 2 in two of its envisioned configurations for intrapoerative ultrasound. Force sensing and basic robot control has been integrated into the robot. In the future, the robot's performance will be assessed, both in terms of its usefulness as a stand alone tool for intraoperative ultrasound, as well as in a variety of cooperative configurations.

\section{Acknowledgments}

The authors gratefully acknowledge the support of the National Science Foundation Engineering Research Center Grant \#EEC9731478. We also thank Dr. Sheila Sheth and Robert De Jong for supplying ultrasound equipment and assistance. In addition, we thank Louis L. Whitcomb for his input over the course of the project.

\section{References}

1) S. E. Salcdean, G. Bell, S Bachmann, W. -H. Zhu, P. Abolmaesumi, and P. D. Lawrence, "Robot-assisted diagnostic ultrasound - design and feasibility experiment," MICCAI '99, UK, 1999

2) E. Degoulange, L. Urbain, P. Caron, S. Boudet, J. Gariepy, J.-L. Megnien, F. Pierrot, and E. Dombre, "HIPPOCRATE: an intrinsically safe robot for medical applications," Proc. IEEE/RSJ Int. Conf. on Intelligent Robots and Systems, IROS'98, Victoria, pp. 959964,1998

3) Stoianovici, D., Whitcomb, L.L., Anderson, J.H., Taylor, R.H., Kavoussi L.R, “A Modular Surgical Robotic System for Image Guided Percutaneous Procedures," Lecture Notes in Computer Science, Springer-Verlga, Vol. 1496, Pp. 404-410, 1998

4) Stoianovici D, Kavoussi LR: Ball-Worm Transmission, (1999), Regular U.S. utility and PCT application filled by the Johns Hopkins University (\#DM-3512) 